\title{
Threshold of the extent of resection for WHO Grade III gliomas: retrospective volumetric analysis of 122 cases using intraoperative MRI
}

\author{
Yu Fujii, MD,1-3 Yoshihiro Muragaki, MD, PhD, 1,2 Takashi Maruyama, MD, PhD, $, 1,2$ \\ Masayuki Nitta, MD, PhD, ${ }^{1,2}$ Taiichi Saito, MD, PhD, ${ }^{1}$ Soko Ikuta, PhD, ${ }^{2}$ Hiroshi Iseki, MD, PhD, ${ }^{2}$ \\ Kazuhiro Hongo, MD, PhD, ${ }^{3}$ and Takakazu Kawamata, MD, PhD' \\ ${ }^{1}$ Department of Neurosurgery and ${ }^{2}$ Faculty of Advanced Techno-Surgery, Institute of Advanced Biomedical Engineering and \\ Science, Graduate School of Medicine, Tokyo Women's Medical University, Tokyo; and 'Department of Neurosurgery, Shinshu \\ University School of Medicine, Matsumoto, Japan
}

\begin{abstract}
OBJECTIVE WHO Grade III gliomas are relatively rare and treated with multiple modalities such as surgery, chemotherapy, and radiotherapy. The impact of the extent of resection (EOR) on improving survival in patients with this tumor type is unclear. Moreover, because of the heterogeneous radiological appearance of Grade III gliomas, the MRI sequence that best correlates with tumor volume is unknown. In the present retrospective study, the authors evaluated the prognostic significance of EOR.
\end{abstract}

METHODS Clinical and radiological data from 122 patients with newly diagnosed WHO Grade III gliomas who had undergone intraoperative MRI-guided resection at a single institution between March 2000 and December 2011 were analyzed retrospectively. Patients were divided into 2 groups by histological subtype: 81 patients had anaplastic astrocytoma (AA) or anaplastic oligoastrocytoma (AOA), and 41 patients had anaplastic oligodendroglioma (AO). EOR was calculated using pre- and postoperative T2-weighted and contrast-enhanced T1-weighted MR images. Univariate and multivariate analyses were performed to evaluate the prognostic significance of EOR on overall survival (OS).

RESULTS The 5-, 8-, and 10-year OS rates for all patients were $74.28 \%, 70.59 \%$, and $65.88 \%$, respectively. The 5and 8 -year OS rates for patients with AA and AOA were $72.2 \%$ and $67.2 \%$, respectively, and the 10 -year OS rate was $62.0 \%$. On the other hand, the 5- and 8-year OS rates for patients with AO were $79.0 \%$ and $79.0 \%$; the 10 -year OS rate is not yet available. The median pre- and postoperative T2-weighted high-signal intensity volumes were $56.1 \mathrm{~cm}^{3}(\mathrm{range}$ $1.3-268 \mathrm{~cm}^{3}$ ) and $5.9 \mathrm{~cm}^{3}$ (range 0-180 $\mathrm{cm}^{3}$ ), respectively. The median EOR of T2-weighted high-signal intensity lesions (T2-EOR) and contrast-enhanced T1-weighted lesions were $88.8 \%$ (range $0.3 \%-100 \%$ ) and $100 \%$ (range $34.0 \%$ $100 \%$ ), respectively. A significant survival advantage was associated with resection of $53 \%$ or more of the preoperative T2-weighted high-signal intensity volume in patients with $\mathrm{AA}$ and $\mathrm{AOA}$, but not in patients with $\mathrm{AO}$. Univariate analysis showed that preoperative Karnofsky Performance Scale score $(p=0.0019)$, isocitrate dehydrogenase 1 (IDH1) mutation $(p=0.0008)$, and T2-EOR $(p=0.0208)$ were significant prognostic factors for survival in patients with AA and AOA. Multivariate analysis demonstrated that T2-EOR (HR 3.28; 95\% Cl 1.22-8.81; $p=0.0192$ ) and IDH1 mutation (HR 3.90; 95\% $\mathrm{Cl} 1.53-10.75 ; p=0.0044$ ) were predictive of survival in patients with $A A$ and $A O A$.

CONCLUSIONS T2-EOR was one of the most important prognostic factors for patients with AA and AOA. A significant survival advantage was associated with resection of $53 \%$ or more of the preoperative T2-weighted high-signal intensity volume in patients with $A A$ and $A O A$.

https://thejns.org/doi/abs/10.3171/2017.3.JNS162383

KEY WORDS extent of resection; WHO Grade III glioma; survival; volumetric analysis; intraoperative MRI; removal rate; oncology 
$\mathrm{W}$ HO Grade III gliomas are categorized as anaplastic astrocytoma (AA), anaplastic oligoastrocytoma (AOA), and anaplastic oligodendroglioma (AO) in the 2007 WHO Classification of Tumours of the Central Nervous System. ${ }^{14}$ WHO Grade III gliomas are uncommon neoplasms: AA represents $1.7 \%$ and $\mathrm{AO}$ represents $0.5 \%$ of primary brain tumors. ${ }^{21}$ Microsurgical resection is one of the main treatment options for gliomas. Emerging evidence suggests that more extensive resection of the tumor leads to better prognosis for all grades of gliomas. Although the number of studies on the extent of resection (EOR) of WHO Grade II and IV gliomas is increasing, few reports on WHO Grade III gliomas have been published, especially regarding the use of volumetric analysis. ${ }^{9,12,13,18,28,29,32}$ Because of the relatively lower prevalence of WHO Grade III gliomas, previous studies often merged such lesions with the much more common WHO Grade IV gliomas (glioblastoma multiforme) and considered them all "high-grade gliomas." 28

In this study, we focused only on patients with WHO Grade III gliomas and conducted volumetric analysis using intraoperative MRI. In previous reports, tumor volume was calculated with T2-weighted ${ }^{18}$ or FLAIR images $^{32}$ of WHO Grade II gliomas and contrast-enhanced T1-weighted images of WHO Grade IV gliomas.12,13,29 However, the literature is sparse regarding which MRI sequence best correlates with tumor volume in WHO Grade III gliomas.

Despite combined multimodal treatment, including resection and chemoradiotherapy, WHO Grade III gliomas still have a poor prognosis. However, overall survival (OS) differs among histological subtypes. AO has a better prognosis than AA: the 5- and 10-year OS rates for AA and $\mathrm{AO}$ are $27.9 \%$ and $19.8 \%$, and $52.5 \%$ and $38.9 \%$, respectively. ${ }^{21} \mathrm{AOA}$ has an intermediate prognosis between AA and $\mathrm{AO} .{ }^{31}$ Therefore, in this study, patients were divided into 2 groups according to the presence or absence of an astrocytic component: patients with AA and AOA were in the one group, and patients with $\mathrm{AO}$ were in another group.

The present retrospective volumetric analysis assessed the influence of EOR on outcome, including OS in patients with WHO Grade III gliomas, and attempted to establish a threshold for EOR.

\section{Methods}

\section{Patient Population}

Clinical and radiological data of consecutive patients with newly diagnosed WHO Grade III gliomas who were treated between March 2000 and December 2011 were retrospectively extracted from our institutional database. Data for a total of 164 patients were initially collected. Twelve patients with other histological subtypes (neurofibromatosis Type 1, anaplastic ependymoma, and gliomatosis cerebri), 3 patients with multiple lesions, and 27 patients who had no or insufficient intraoperative MRI data were excluded. Finally, 122 patients (76 male and 46 female patients) were included in the study. Tumor grading and pathological diagnosis were confirmed based on the 2007 WHO Classification of Tumours of the Central Ner- vous System. Patients were divided into 2 groups: AA and AOA (81 patients) or AO (41 patients). Clinical data were collected from the medical records and through telephone interviews. This study was approved by the institutional review board of Tokyo Women's Medical University. The requirement for informed consent of the participants was waived.

\section{Image Acquisition}

MR images were acquired using a 0.3-T intraoperative open MRI system (AIRIS II, Hitachi Medical Co.). Intraoperative MRI was performed at least 2 times, before and after resection of the tumor. Additional MRI was performed at intervals determined by the surgeon throughout surgery, if necessary. In this article, pre- and postoperative tumor volumes are described as the volumes measured on intraoperative MRI before and after resection, respectively. With respect to tumor location, the eloquent region was defined as 1 or more of the following areas: internal capsule, basal ganglia, language cortex, sensory cortex, motor cortex, visual center, thalamus, hypothalamus, brainstem, and dentate nucleus. ${ }^{12,32}$

\section{Treatment}

Since 2000, our institution has performed "information-guided surgery" using intraoperative MRI and updated navigation, which enables maximum resection of gliomas. ${ }^{8,17}$ Maximum resection of T2-weighted high-signal intensity and contrast-enhanced T1-weighted lesions, if any, was performed using an updated navigation system and intraoperative MRI. Intraoperative monitoring such as motor and somatosensory evoked potentials was used routinely. Intraoperative mapping techniques such as awake speech/language mapping and direct cortical motor stimulation were also used, mainly in patients with lesions in the eloquent areas. Surgery was performed before chemotherapy and radiation therapy (RT) in all cases.

Postoperative treatments were conducted in almost all cases, except 5 patients who did not undergo chemotherapy and 3 patients who did not undergo RT. The nimustine hydrochloride-based regimen was mainly used for adjuvant chemotherapy. Ten patients were treated with temozolomide. One hundred fourteen patients received extended local fractionated RT (range 46-60 Gy), 2 patients received local radiotherapeutic methods, and 1 patient died while receiving RT.

\section{Volumetric Analysis}

Manual segmentation was performed with region-of-interest analysis to the measure tumor volumes $\left(\mathrm{cm}^{3}\right)$ based on axial T2-weighted and contrast-enhanced T1-weighted intraoperative MR images (Fig. 1). Tumor volumes were calculated by exporting DICOM images from intraoperative MRI to Leksell GammaPlan software (Elekta). EOR was defined as follows: (preoperative tumor volume postoperative tumor volume)/preoperative tumor volume. EOR and residual tumor volume were calculated for both T2-weighted high-signal intensity lesions and contrastenhanced T1-weighted lesions and dichotomized to evaluate the threshold. 

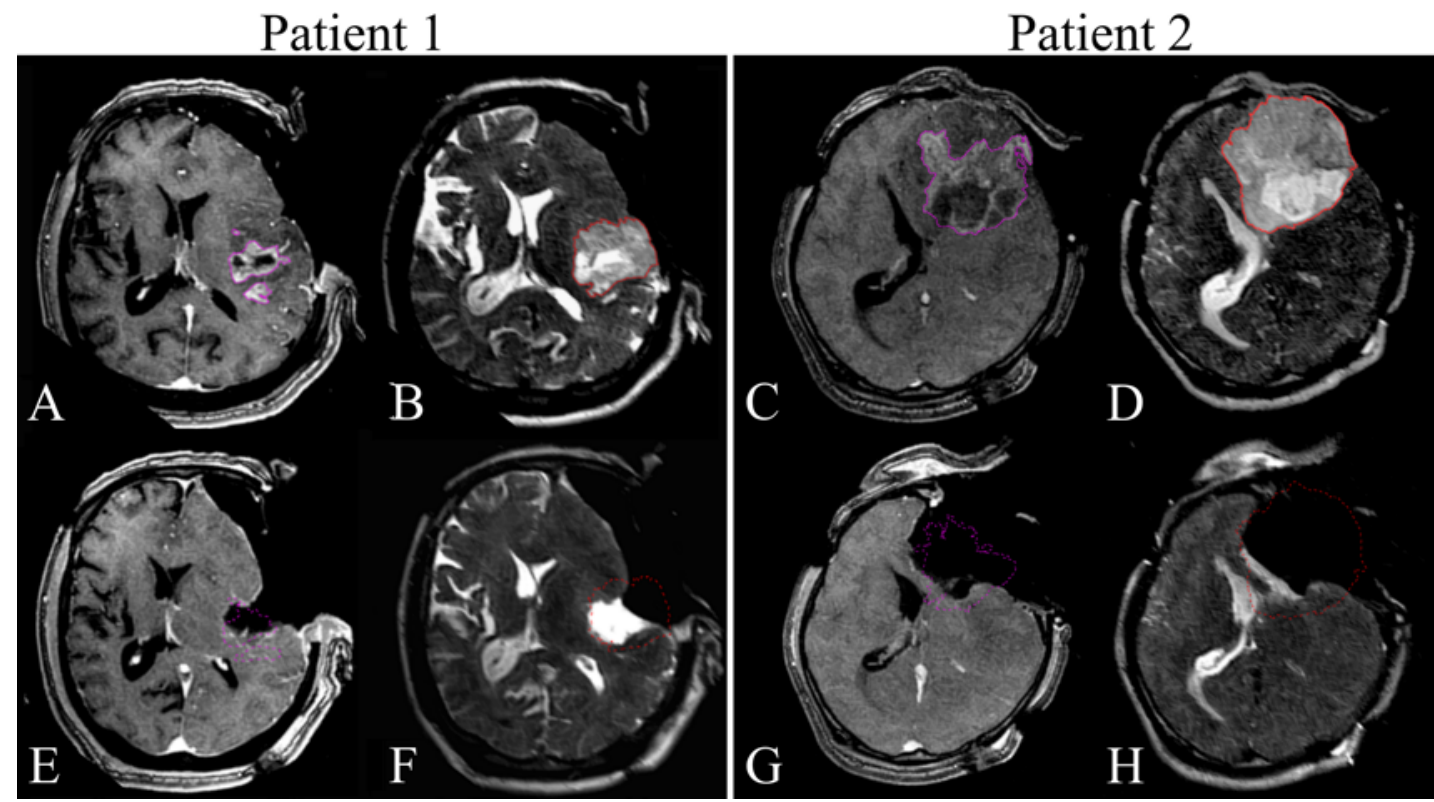

FIG. 1. Representative images of volumetric measurements in 2 patients. Segmentation of axial contrast-enhanced T1-weighted and T2-weighted volumes on preoperative (A and B [Patient 1]; $\mathbf{C}$ and $\mathbf{D}$ [Patient 2]) and (E and $\mathbf{F}$ [Patient 1]; $\mathbf{G}$ and $\mathbf{H}$ [Patient 2]) MR images. This overlay shows the preoperative contrast-enhanced volume (purple) and preoperative T2-weighted hyperintense volume (red).

\section{Statistical Analysis}

OS-defined as the time between initial surgery and death-was assessed. The probability of OS was estimated using the Kaplan-Meier method. The log-rank test was used to evaluate the importance of prognostic factors that may affect survival. Cox proportional hazards modeling was used for the univariate and multivariate analyses of OS. HRs and 95\% CIs are reported. Two-tailed probability values are used, and $p<0.05$ is considered statistically significant. To identify the threshold, EOR and residual tumor were divided into 2 groups. All statistical analyses were performed using JMP statistical software (SAS Inc.).

\section{Results}

\section{Patient Characteristics}

Patient characteristics are described in Table 1. The median age was 40 years (range 17-78 years), and the median preoperative Karnofsky Performance Scale (KPS) score was 100 (range 40-100). The numbers of patients with AA, AOA, and AO were 42 (34.4\%), 39 (32.0\%), and 41 (33.6\%), respectively. The most common area of tumor location was the frontal lobe (71 patients [56.6\%]). Forty-eight percent of tumors were located in an eloquent region of the brain. Twenty percent of tumors were deep lesions, which were defined as those located in the insula, thalamus, basal ganglia, or posterior fossa, as described by Lacroix et al. ${ }^{12}$ The median MIB-1 labeling index was 12.9\% (range 3.5\%59.2\%). RT was administered to 119 patients (97.5\%), and nimustine hydrochloride-based chemotherapy was given to 116 patients $(95.9 \%)$. Isocitrate dehydrogenase 1 (IDHI) mutation (R132S) was found in $82(69.5 \%)$ of 118 patients analyzed. Codeletion of $1 \mathrm{p} / 19 \mathrm{q}$ was detected in 44 (44.4\%) of 99 patients investigated. The 5- and 8-year OS rates for all patients were $74.28 \%$ and $70.59 \%$, respectively, and the 10 -year OS rate was $65.88 \%$ (Fig. 2A). The 5- and 8-year $\mathrm{OS}$ rates for patients with AA and AOA were $72.2 \%$ and $67.2 \%$, respectively, and the 10 -year OS rate was $62.0 \%$. On the other hand, the 5- and 8-year OS rates for patients with $\mathrm{AO}$ were $79.0 \%$ and $79.0 \%$; the 10 -year OS rate is not yet available (Fig. 2B). No statistically significant difference in the OS rates was found between the 2 groups according to tumor subtype $(p=0.45)$. The median follow-up period was 44 months (range $1.5-150$ months).

\section{Preoperative and Postoperative Tumor Volumes and EOR}

Tumor volumes and EOR are summarized in Table 2. The median and mean volumes of preoperative T2weighted high-signal intensity lesions were $56.1 \mathrm{~cm}^{3}$ (range 1.3-268.3 $\mathrm{cm}^{3}$ ) and $71.9 \mathrm{~cm}^{3}$, respectively. The median and mean volumes of postoperative T2-weighted high-signal intensity lesions were $5.9 \mathrm{~cm}^{3}$ (range $0-180.5$ $\mathrm{cm}^{3}$ ) and $19.0 \mathrm{~cm}^{3}$, respectively. Fifty-one (41.8\%) of the 122 patients exhibited contrast enhancement on T1weighted images. The median and mean volumes of preoperative contrast-enhanced T1-weighted lesions were 4.2 $\mathrm{cm}^{3}$ (range $0.1-77.6 \mathrm{~cm}^{3}$ ) and $10.6 \mathrm{~cm}^{3}$, respectively. The median and mean volumes of the postoperative contrastenhanced T1-weighted lesions were $0 \mathrm{~cm}^{3}$ (range 0-10.0 $\mathrm{cm}^{3}$ ) and $0.7 \mathrm{~cm}^{3}$, respectively. All patients underwent intraoperative MRI-guided microsurgical resection. Median and mean EOR of T2-weighted high-signal intensity lesions (T2-EOR) were $88.8 \%$ (range $0.3 \%-100 \%$ ) and $77.7 \%$, respectively (Fig. 3). The median and mean EOR of contrast-enhanced T1-weighted lesions were $100 \%$ (range $34.0 \%-100 \%$ ) and $96.0 \%$, respectively. 
TABLE 1. Clinical and tumor characteristics of 122 patients with WHO Grade III gliomas

\begin{tabular}{|c|c|c|c|}
\hline \multirow[b]{2}{*}{ Characteristic } & \multicolumn{2}{|c|}{ Tumor Group } & \multirow[b]{2}{*}{$\mathrm{p}$ Value } \\
\hline & $A A+A O A$ & $\mathrm{AO}$ & \\
\hline No. of patients & $81^{*}$ & 41 & \\
\hline Sex & & & 0.171 \\
\hline Male & $47(58.0)$ & $29(70.7)$ & \\
\hline Female & $34(42.0)$ & $12(29.3)$ & \\
\hline Age at diagnosis & & & 0.534 \\
\hline$<50$ yrs & $54(66.7)$ & $25(61.0)$ & \\
\hline$\geq 50 \mathrm{yrs}$ & $27(33.3)$ & $16(39.0)$ & \\
\hline KPS score at diagnosis & & & 0.771 \\
\hline$<80$ & $5(6.2)$ & $2(4.9)$ & \\
\hline 80 & $8(9.9)$ & $2(4.9)$ & \\
\hline 90 & $14(17.3)$ & $12(29.3)$ & \\
\hline 100 & $54(66.6)$ & $25(60.9)$ & \\
\hline Radiotherapy & & & 0.992 \\
\hline Yes & $79(97.5)$ & $40(97.6)$ & \\
\hline No & $2(2.5)$ & $1(2.7)$ & \\
\hline Chemotherapy & & & 0.368 \\
\hline Yes & $76(93.8)$ & $40(97.6)$ & \\
\hline No & $5(6.2)$ & $1(2.7)$ & \\
\hline Side of tumor & & & $0.004 \dagger$ \\
\hline Left & $37(45.7)$ & $31(75.6)$ & \\
\hline Right & $44(54.3)$ & $10(24.4)$ & \\
\hline Tumor location & & & 0.211 \\
\hline Frontal lobe & $45(55.6)$ & $26(63.4)$ & \\
\hline Insula & $7(8.6)$ & $7(17.1)$ & \\
\hline Temporal lobe & $9(11.1)$ & $5(12.2)$ & \\
\hline Parietal lobe & $13(16.0)$ & $3(7.3)$ & \\
\hline Occipital lobe & $3(3.7)$ & 0 & \\
\hline Infratentorial & $4(4.9)$ & 0 & \\
\hline Eloquent tumor location $\ddagger$ & $41(50.6)$ & $18(43.9)$ & \\
\hline p53 status & & & $<0.001 \dagger$ \\
\hline Positive & $43(44.2)$ & $8(20)$ & \\
\hline Negative & $34(55.8)$ & $32(80)$ & \\
\hline Not available & 4 & 1 & \\
\hline IDH1 status & & & 0.141 \\
\hline Mutant & $50(64.9)$ & $32(78.0)$ & \\
\hline Wild type & $27(35.1)$ & $9(22.0)$ & \\
\hline Not available & 4 & 0 & \\
\hline $1 p / 19 q$ status & & & $<0.001 \dagger$ \\
\hline Codeletion & $15(25)$ & $29(74.4)$ & \\
\hline Non-codeletion & $45(75)$ & $10(25.6)$ & \\
\hline Not available & 21 & 2 & \\
\hline Contrast enhancement & & & 0.658 \\
\hline Positive & $35(43.2)$ & $16(39.0)$ & \\
\hline Negative & $46(56.8)$ & $25(61.0)$ & \\
\hline
\end{tabular}

All values are shown as number (\%) of patients.

* Forty-two patients had AA, and 39 patients had AOA.

† Statistically significant at $p<0.05$

$\ddagger$ Tumor was located in 1 or more of the following regions: internal capsule, basal ganglia, language cortex, sensory cortex, motor cortex, visual center, thalamus, hypothalamus, brainstem, or dentate nucleus.

\section{Univariate and Multivariate Analysis}

T2-EOR was statistically significant at several cutoff values in the univariate analysis of the 122 patients with AA, AOA, and AO (Table 3). However, a threshold could not be established. Then, T2-EOR for each histological subtype was analyzed. T2-EOR was not statistically significant for OS at any cutoff value in patients with $\mathrm{AO}$. On the other hand, in patients with AA and AOA, resection of $53 \%$ or more of the preoperative T2-weighted high-signal intensity volume was associated with a significant prognostic benefit (Fig. 4).

As for the EOR results, the residual tumor volume of T2-weighted high-signal intensity lesions (T2-RTV) was statistically significant at several cutoff values in the univariate analysis of the 122 patients with all subtypes, but a threshold was not determined. T2-RTV for each histological subtype was also analyzed. T2-RTV was not statistically significant for OS at any cutoff value in patients with AO. On the other hand, in patients with AA and AOA, T2-RTV of less than $25 \mathrm{~cm}^{3}$ was associated with a significant prognostic benefit. Subsequent univariate and multivariate analyses were conducted in 81 patients with AA and AOA.

Significant $(p<0.05)$ parameters for OS in the univariate analysis included preoperative KPS score, $I D H 1 \mathrm{mu}-$ tation, and T2-EOR (Table 4). The multivariate analysis was performed using the following parameters: age, KPS score, IDHI mutation, and T2-EOR or T2-RTV (Table 5). The parameters identified as significant for OS were T2-EOR (HR 3.58; 95\% CI 1.32-9.37; $\mathrm{p}=0.014$ ) and IDH1 mutation (HR 4.15; 95\% CI 1.63-11.42; $\mathrm{p}=0.003$ ). T2-RTV did not show a statistically significant benefit for OS in the multivariate analysis (HR 1.99; 95\% CI 0.18$1.51 ; \mathrm{p}=0.211$ ).

\section{Discussion}

Previous reports that specifically focused on WHO Grade III gliomas are limited to only a few series. The present study investigated the prognostic significance of EOR in patients with WHO Grade III gliomas. T2-EOR showed a strong correlation with survival in patients with $\mathrm{AA}$ and $\mathrm{AOA}$ in the univariate and multivariate analyses. In those patients, T2-EOR $\geq 53 \%$ was associated with improved survival.

Although some studies such as that by Tortosa et al. did not find an association between EOR and survival, ${ }^{25,33}$ several other studies demonstrated the prognostic significance of EOR in patients with Grade III gliomas. In those studies, patients with AA were mainly studied, and patients with AOA were often excluded. ${ }^{19,20,22,31}$ According to Sanai and Berger's review, 4 volumetric studies of high-grade gliomas have been published, including only 1 volumetric study of AA that was performed using conventional MRI between 1990 and $2008 .{ }^{28}$ In the present study, all subtypes of WHO Grade III gliomas were analyzed with the volumetric method using intraoperative MRI.

In previous reports, the residual volume of contrastenhancing lesions and the pattern of contrast enhancement were associated with decreased survival., ${ }^{4,92,35}$ To the best of our knowledge, Pessina et al. were the first to 

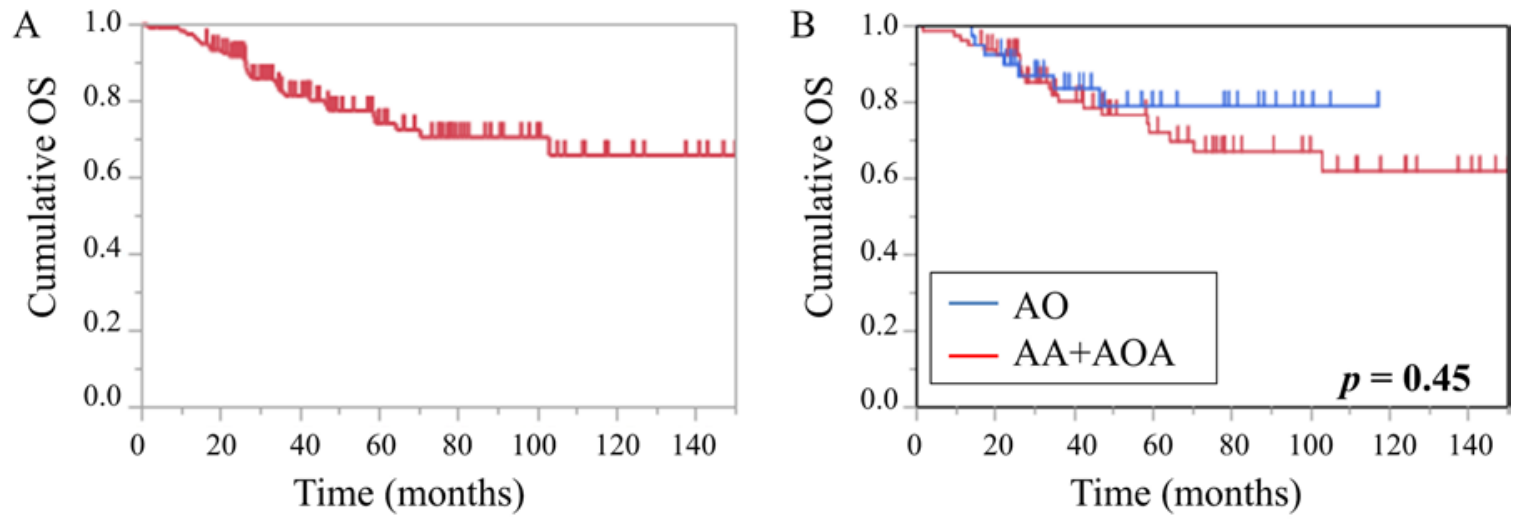

Number at risk

$\begin{array}{lllllllll}122 & 110 & 69 & 45 & 27 & 16 & 8 & 4 & \mathrm{AO}\end{array}$

Number at risk

$\begin{array}{lllllrrrr}\text { AO } & 41 & 37 & 22 & 13 & 9 & 3 & 0 & 0 \\ \text { AA+AOA } & 81 & 74 & 47 & 32 & 18 & 13 & 8 & 4\end{array}$

FIG. 2. Kaplan-Meier OS curves for all 122 newly diagnosed patients with WHO Grade III gliomas (A) and patients divided into 2 groups by histological subtype (B).

demonstrate that an EOR threshold $>76 \%$ and a residual tumor volume $<3 \mathrm{~cm}^{3}$ were significant factors associated with improved survival in WHO Grade III gliomas. ${ }^{24} \mathrm{Sev}-$ eral differences are present between their study and ours. First, EOR in their report was a mixed value because it was calculated using contrast-enhanced T1-weighted images for contrast-enhancing tumors and FLAIR images for nonenhancing tumors. In contrast, EOR in our study was only calculated for T2-weighted hyperintense lesions regardless of whether contrast enhancement was present. Because WHO Grade III gliomas have a heterogeneous radiological appearance, only 50\%-70\% show contrast enhancement. Moreover, contrast-enhancing lesions may not accurately correlate with tumor burden because an infiltrative tumor could present in the surrounding T2weighted hyperintense area. Second, almost half of the cases in their study were those of malignant transformation. These facts may result in a lower EOR threshold in our study compared with their study. Our study is the first report to establish an EOR threshold, which was only calculated for T2-weighted hyperintense lesions in primary WHO Grade III gliomas.

Regarding the MRI sequence and threshold of EOR, Smith et al. suggested that an improved outcome is predicted by the greater resection $(\geq 90 \%)$ of FLAIR hyperintense lesions in WHO Grade II gliomas. ${ }^{32} \mathrm{Li}$ et al. reported that the additional resection of $\geq 53.21 \%$ of FLAIR hyperintense lesions, which surround contrast-enhanced lesions, may be associated with better prognosis and established this threshold for WHO Grade IV gliomas. ${ }^{12,13}$ Our data are complementary to these studies and establish the threshold of EOR for T2-weighted/FLAIR hyperintense lesions of all grades of gliomas.

With respect to residual tumor volume, some reports contend that residual tumor volume is the more significant predictor of survival compared with EOR.,24 Moreover, Pessina et al. ${ }^{24}$ concluded that the presence of contrastenhancing residual tumor volume was an unfavorable prognostic factor compared with the residual tumor volume of WHO Grade III gliomas on FLAIR images. In our
TABLE 2. Summary of preoperative and postoperative tumor volumes and EOR

\begin{tabular}{|c|c|c|}
\hline Variable & $\begin{array}{l}\text { T2-Weighted } \\
\text { Imaging }\end{array}$ & $\begin{array}{l}\text { Contrast-Enhanced } \\
\text { T1-Weighted } \\
\text { Imaging }\end{array}$ \\
\hline No. of patients & 122 & 51 \\
\hline \multicolumn{3}{|c|}{ Preoperative tumor volume, $\mathrm{cm}^{3}$} \\
\hline 0 & 0 & 0 \\
\hline $1-24$ & 25 & 46 \\
\hline $25-50$ & 29 & 2 \\
\hline $51-100$ & 38 & 3 \\
\hline $101-300$ & 30 & 0 \\
\hline Median & 56.1 & 4.2 \\
\hline Mean & 71.9 & 10.6 \\
\hline Range & $1.3-268.3$ & $0.1-77.6$ \\
\hline \multicolumn{3}{|c|}{ Postoperative tumor volume, $\mathrm{cm}^{3}$} \\
\hline 0 & 17 & 38 \\
\hline $0.1-10$ & 56 & 12 \\
\hline $10.1-50.0$ & 43 & 1 \\
\hline $50.1-200.0$ & 6 & 0 \\
\hline Median & 5.9 & 0 \\
\hline Mean & 19.0 & 0.7 \\
\hline Range & $0-180.5$ & $0-10.0$ \\
\hline \multicolumn{3}{|l|}{ EOR, \% } \\
\hline $0-40$ & 12 & 1 \\
\hline $41-60$ & 15 & 1 \\
\hline $61-89$ & 38 & 3 \\
\hline $90-99$ & 40 & 8 \\
\hline 100 & 17 & 38 \\
\hline Median & 88.8 & 100 \\
\hline Mean & 77.7 & 96.0 \\
\hline Range & $0.3-100$ & $34.0-100$ \\
\hline
\end{tabular}




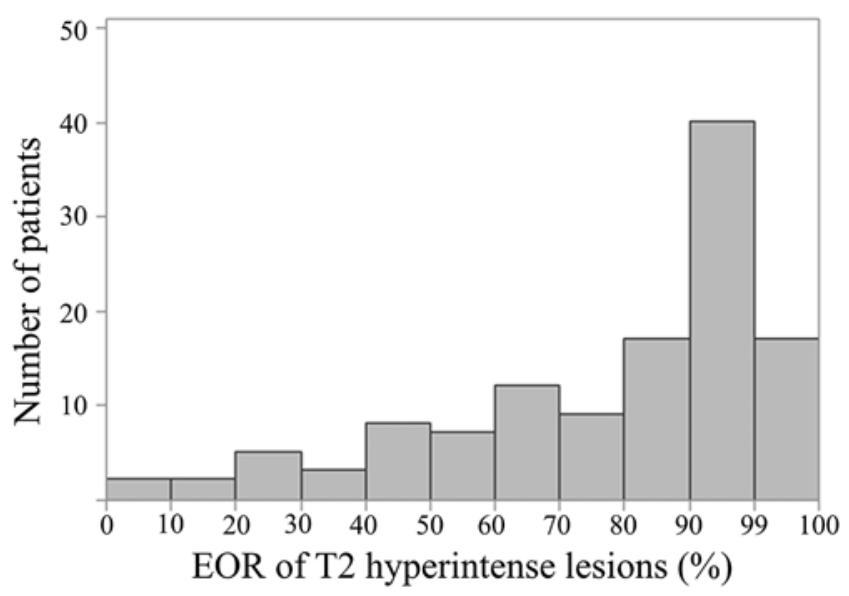

FIG. 3. Bar graph showing the distribution of 122 patients with WHO Grade III gliomas according to the different EORs of T2-weighted hyperintense lesions (median 88.8\%; range 0.3\%-100\%).

series, although T2-RTV $<25 \mathrm{~cm}^{3}$ was associated with a significant prognostic benefit in patients with AA and AOA in the univariate analysis, the multivariate analysis indicated that EOR was a stronger predictor than T2-RTV. This discrepancy between previous reports and ours may arise from 2 reasons. First, as mentioned above, the MRI sequence used to measure EOR in our study was different from that used in previous studies. Second, our study involved biases toward a high EOR distribution and low T2-RTV distribution. This biased distribution was caused by extensive resection, which may be due to the use of intraoperative MRI, and it was also seen in patients with glioblastoma in our institute. ${ }^{5}$

In the present study, we calculated T2-EOR using intraoperative MRI. This sequence and timing of MRI may contribute to accurate EOR of the tumor burden. This is because early (mainly defined as $<48$ hours) postoperative MRI, which was often used for calculation in previous studies, $3,4,7,9,13,24,29$ overestimates the residual tumor volume in either T2-weighted sequences or FLAIR-weighted sequences. $^{2}$ This overestimation may be due to edema or contusion of the brain tissue surrounding the resection cavity and ischemia induced by surgical devascularization. Moreover, according to Pala et al., intraoperative T2-weighted/FLAIR abnormalities showed no significant differences in residual tumor volumes compared with late postoperative images, which were obtained 3-4 months after surgery. ${ }^{23}$ Thus, the use of intraoperative MRI after final resection of the tumor may correct the overestimation and define tumor borders more precisely than conventional early postoperative MRI.

Intraoperative MRI has another benefit because it contributes to greater EOR during glioma surgery. This advantage was shown by Kubben et al. in a systematic review ${ }^{11}$ and by Senft et al. in a randomized controlled trial. ${ }^{30}$ Whereas Senft et al. focused on contrast-enhancing gliomas that were mainly WHO Grade IV, Mohammadi et al. ${ }^{16}$ evaluated both enhancing and nonenhancing gliomas. They concluded that intraoperative MRI is also useful for nonenhancing gliomas, which mainly consisted of
TABLE 3. OS shown according to EOR*

\begin{tabular}{|c|c|c|c|}
\hline T2-EOR, \% & $\mathrm{HR}$ & $95 \% \mathrm{Cl}$ & p Value \\
\hline \multicolumn{4}{|c|}{ All WHO Grade III patients ( $n=122)$} \\
\hline$<24$ vs $\geq 24$ & 3.41 & $0.81-9.80$ & 0.087 \\
\hline$<25$ vs $\geq 25$ & 4.40 & $1.28-11.50$ & $0.022 \dagger$ \\
\hline$<30$ vs $\geq 30$ & 4.51 & $1.50-11.14$ & $0.010 \dagger$ \\
\hline$<40$ vs $\geq 40$ & 2.24 & $0.75-5.46$ & 0.136 \\
\hline$<50$ vs $\geq 50$ & 2.04 & $0.80-4.58$ & 0.127 \\
\hline$<60$ vs $\geq 60$ & 2.80 & $1.27-5.92$ & $0.012 \dagger$ \\
\hline$<89$ vs $\geq 89$ & 2.28 & $1.06-5.30$ & $0.034 \dagger$ \\
\hline$<90$ vs $\geq 90$ & 1.99 & $0.93-4.63$ & 0.078 \\
\hline$<92$ vs $\geq 92$ & 1.96 & $0.90-4.74$ & 0.094 \\
\hline$<93$ vs $\geq 93$ & 2.27 & $1.01-5.78$ & $0.046 \dagger$ \\
\hline$<94$ vs $\geq 94$ & 2.42 & $1.04-6.58$ & $0.039 \dagger$ \\
\hline$<95$ vs $\geq 95$ & 2.29 & $0.99-6.24$ & 0.053 \\
\hline \multicolumn{4}{|l|}{$A A+A O A$ patients $(n=81)$} \\
\hline$<24$ vs $\geq 24$ & 1.98 & $0.10-9.07$ & 0.546 \\
\hline$<25$ vs $\geq 25$ & 3.64 & $0.58-12.71$ & 0.143 \\
\hline$<30$ vs $\geq 30$ & 3.93 & $0.90-11.99$ & 0.065 \\
\hline$<40$ vs $\geq 40$ & 1.42 & $0.33-4.20$ & 0.593 \\
\hline$<50$ vs $\geq 50$ & 1.94 & $0.63-4.95$ & 0.226 \\
\hline$<52$ vs $\geq 52$ & 2.51 & $0.95-6.05$ & 0.062 \\
\hline$<53$ vs $\geq 53$ & 3.03 & $1.20-7.21$ & $0.021 \dagger$ \\
\hline$<60$ vs $\geq 60$ & 2.88 & $1.17-6.82$ & $0.022 \dagger$ \\
\hline$<70$ vs $\geq 70$ & 2.78 & $1.18-6.84$ & $0.020 \dagger$ \\
\hline$<80$ vs $\geq 80$ & 3.50 & $1.45-9.24$ & $0.005 \dagger$ \\
\hline$<90$ vs $\geq 90$ & 2.69 & $1.09-7.56$ & $0.031 \dagger$ \\
\hline$<95$ vs $\geq 95$ & 4.61 & $1.56-19.68$ & $0.004 \dagger$ \\
\hline$<98$ vs $\geq 98$ & 3.77 & $1.09-23.66$ & $0.034 \dagger$ \\
\hline \multicolumn{4}{|l|}{ AO patients $(n=41)$} \\
\hline$<40$ vs $\geq 40$ & 6.99 & $0.99-33.00$ & 0.051 \\
\hline$<50$ vs $\geq 50$ & 2.49 & $0.36-11.72$ & 0.313 \\
\hline$<70$ vs $\geq 70$ & 2.99 & $0.58-13.72$ & 0.174 \\
\hline$<80$ vs $\geq 80$ & 1.38 & $0.27-6.29$ & 0.680 \\
\hline$<90$ vs $\geq 90$ & 1.01 & $0.22-5.12$ & 0.994 \\
\hline$<93$ vs $\geq 93$ & 0.66 & $0.14-3.39$ & 0.595 \\
\hline$<95$ vs $\geq 95$ & 0.50 & $0.11-2.56$ & 0.379 \\
\hline$<98$ vs $\geq 98$ & 0.69 & $0.15-4.84$ & 0.665 \\
\hline
\end{tabular}

low-grade gliomas.${ }^{16}$ Our data are compatible with these results, showing a high percentage of EOR in both enhancing and nonenhancing tumors: the median T2-EOR and EOR of contrast-enhanced T1-weighted lesions were $88.8 \%$ and $100 \%$, respectively.

Postoperative therapy, including chemotherapy and RT, is variable among institutions. As mentioned above, almost all patients with WHO Grade III gliomas at our institution received uniform postoperative chemotherapy and RT. This fact may result in less variability and a greater effect on EOR.

In some previous reports, $\mathrm{AO}$ and $\mathrm{AOA}$ were grouped 


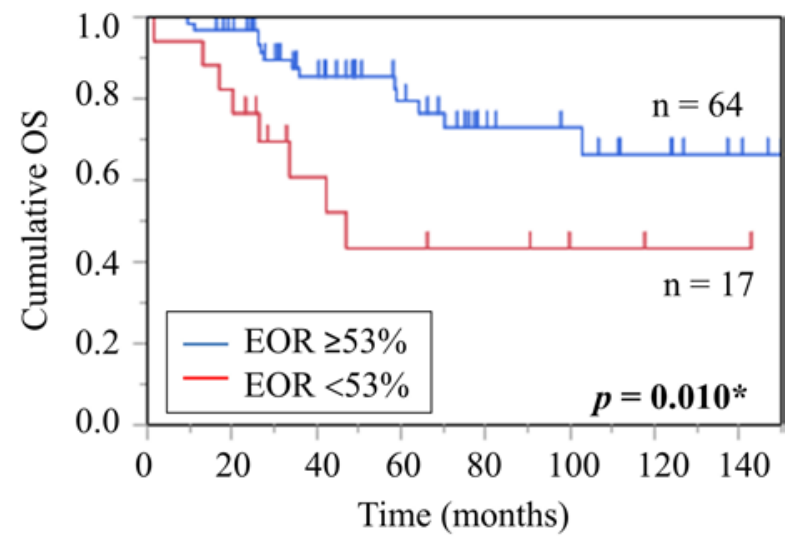

Number at risk

$\begin{array}{rrrrrrrrr}\mathrm{EOR} \geq 53 \% & 64 & 59 & 40 & 27 & 14 & 11 & 7 & 3 \\ \mathrm{EOR}<53 \% & 17 & 14 & 7 & 5 & 4 & 2 & 1 & 1\end{array}$

FIG. 4. Kaplan-Meier OS curves in relation to the EOR threshold for patients with AA and AO. EOR is correlated with patient outcome. Asterisk indicates a significant $p$ value.

as "glioma with an oligodendroglial component."' However, some studies comparing the prognosis of $\mathrm{AO}$ with AOA showed different results, with AOA having an intermediate prognosis between $\mathrm{AA}$ and $\mathrm{AO} .{ }^{31}$ Thus, grouping $\mathrm{AA}$ and AOA as "glioma with an astrocytic component" seems reasonable. In the present study, the 5- and 8-year OS rates for patients with AA and AOA were $72.2 \%$ and $67.2 \%$, and the 10 -year OS rate was $62.0 \%$. On the other hand, the 5- and 8-year OS rates for patients with AO were $79.0 \%$ and $79.0 \%$; the 10 -year OS rate is not yet available. Although we found no statistically significant difference between the 2 groups, patients with $\mathrm{AO}$ showed a trend toward improved OS compared with patients with AA and AOA.

In the univariate analysis, T2-EOR was not significantly different in patients with $\mathrm{AO}$ at any cutoff value. This result may be explained by the observation that $72.5 \%$ of patients with $\mathrm{AO}$ in our study showed $1 \mathrm{p}$ and $19 \mathrm{q}$ codeletions, which are correlated with response to chemotherapy. Moreover, 6 of 7 deceased patients with AO did not show this genetic change. Regarding the limited follow-up period, chemotherapy seemed to be more strongly associated with survival than resection. Even longer follow-up times will be necessary for this analysis. In the present study, $\mathrm{T} 2-\mathrm{EOR} \geq 53 \%$ was associated with improved survival in patients with AA and AOA. However, after subdividing these patients into 2 cohorts (42 patients with AA and 39 patients with AOA), T2-EOR was statistically significant at some cutoff values in both cohorts and a threshold was not established (data not shown). This may be due to the relatively small number of patients with each subtype and the non-uniform distribution of T2-EOR, which is biased toward a high percentage in our series.

IDH1 mutations are present in $60 \%-70 \%$ of WHO Grade III gliomas and associated with a better overall prognosis compared with wild-type $I D H I$ tumors. ${ }^{10}$ In addition, IDH1 mutant malignant astrocytomas are associated with extensive resection and better prognosis. ${ }^{1}$ Our
TABLE 4. Univariate analysis of the survival outcomes for 81 patients with $A A$ and $A O A$

\begin{tabular}{lccc}
\hline \multicolumn{1}{c}{ Factor } & HR & $95 \% \mathrm{Cl}$ & p Value \\
\hline Age & & & \\
\hline$\geq 50$ vs $<50$ yrs & 2.12 & $0.88-5.06$ & 0.091 \\
\hline $\begin{array}{c}\text { Sex } \\
\text { Female vs male }\end{array}$ & 1.07 & $0.44-2.53$ & 0.880 \\
\hline KPS score & & & \\
\hline$<80$ vs $\geq 80$ & 6.84 & $2.23-17.60$ & $0.002^{*}$ \\
\hline Tumor subtype & & & \\
\hline AA vs AOA & 1.64 & $0.68-4.34$ & 0.276 \\
\hline Contrast enhancement & & & \\
\hline Positive vs negative & 1.32 & $0.55-3.13$ & 0.529 \\
\hline Chemotherapy & & & \\
\hline No vs yes & 0.57 & $0.03-2.75$ & 0.551 \\
\hline MIB-1 index & & & \\
\hline$\geq 15 \%$ vs $<15 \%$ & 2.40 & $0.99-5.96$ & 0.052 \\
\hline IDH1 status & & & \\
\hline Wild type vs mutant & 4.82 & $1.92-13.07$ & $0.001^{*}$ \\
\hline EOR & & & $0.021^{*}$ \\
\hline$<53 \%$ vs $\geq 53 \%$ & 3.03 & $1.20-7.21$ & \\
\hline T2-RTV & & & $0.025^{*}$ \\
\hline$<25$ vs $\geq 25$ cm ${ }^{3}$ & 2.82 & $1.15-6.67$ & \\
\hline
\end{tabular}

* Statistically significant at $p<0.05$.

data are compatible with these reports, although few studies have shown the prognostic significance of IDHI mutation in a multivariate analysis.

Given the present results, we propose a surgical strategy for WHO Grade III gliomas. In our series, T2-EOR showed a strong correlation with survival in patients with AA and AOA, and an especially significant advantage was seen with $\mathrm{T} 2$-EOR $\geq 53 \%$. Thus, surgeons should aim for maximum tumor resection if the patient does not have oligodendroglial features as part of their preoperative radiological findings such as calcification on computed tomography ${ }^{27}$ or high uptake on ${ }^{11} \mathrm{C}$-methionine positron emission tomography. ${ }^{26}$

Several limitations of this study should be acknowledged. First, because of the retrospective analysis using intraoperative MRI, selection bias of the patients may be present. This study included few biopsy cases and cases with lower KPS scores. In addition, a few cases were excluded because of inadequate quality of the intraoperative MR images available for review. However, we tried to create a uniform patient population by examining consecutive patients. Although the size of the contrast-enhanced T1weighted lesions in our patients was relatively small compared with previous reports, ${ }^{9}$ the size of the T2-weighted high-signal intensity lesions (median $56 \mathrm{~cm}^{3}$ ), for which we established the threshold of EOR, was larger than other grades of gliomas in previous reports (median $36.6 \mathrm{~cm}^{3}$ for Grade II gliomas; median $30.5 \mathrm{~cm}^{3}$ for Grade IV gliomas). ${ }^{9,32}$ Moreover, in our study, $48 \%$ of tumors were eloquent lesions and $20 \%$ of tumors were deep lesions. These 
TABLE 5. Multivariate analysis of the survival outcomes of 81 patients with $A A$ and $A O A$

\begin{tabular}{llll}
\hline \multicolumn{1}{c}{ Factor } & $\mathrm{HR}$ & $95 \% \mathrm{Cl}$ & $\mathrm{p} \mathrm{Value}$ \\
\hline EOR & & & \\
\hline Age & & & \\
\hline$\geq 50$ vs $<50$ yrs & 1.44 & $0.54-3.76$ & 0.458 \\
\hline KPS score & & & \\
\hline$<80$ vs $\geq 80$ & 3.23 & $0.91-9.06$ & 0.067 \\
\hline IDH1 status & & & \\
\hline Wild type vs mutant & 4.15 & $1.63-11.42$ & $0.003^{*}$ \\
\hline EOR & & & \\
\hline$<53 \%$ vs $\geq 53 \%$ & 3.58 & $1.32-9.37$ & $0.014^{*}$ \\
\hline T2-RTV & & & \\
\hline Age & & & \\
\hline$\quad \geq 50$ vs $<50$ yrs & 1.95 & $0.74-5.05$ & 0.174 \\
\hline KPS score & & & \\
\hline$<80$ vs $\geq 80$ & 2.44 & $0.59-8.65$ & 0.204 \\
\hline IDH1 status & & & \\
\hline Wild type vs mutant & 3.39 & $1.31-9.47$ & $0.012^{*}$ \\
\hline T2-RTV & & & 0.211 \\
\hline$<25$ vs $\geq 25 \mathrm{~cm}^{3}$ & 1.99 & $0.18-1.51$ & \\
\hline
\end{tabular}

* Statistically significant at $p<0.05$.

data are similar to data in previous studies (58\% and $44 \%$ of Grade II and IV eloquent lesions, respectively, ${ }^{12,32}$ and $9 \%$ of Grade IV deep-seated lesions ${ }^{12}$ ). Thus, we can say that we did not intend to select resectable tumors. Generally, randomized control studies are preferred, but such a study would be unethical and impractical. Therefore, only 1 randomized study on EOR that was limited to elderly people was reported.$^{34}$ Second, measuring bias may exist. Because we measured T2-weighted hyperintense lesions in this study, preoperative edema, ischemia, and contusions may have been part of the calculation to some degree. Third, the patients in this study were classified by tumor grade and pathological diagnosis based on the 2007 WHO Classification of Tumours of the Central Nervous System. Because examination of $1 \mathrm{p} / 19 \mathrm{q}$ codeletion began in 2004 at our institution, the total number of patients was smaller and not sufficient for reanalysis of the data using the latest WHO classification. Instead, we subdivided the patients into 2 cohorts: those with and those without a "pure" oligodendroglial tumor. We presumed these "pure" oligodendroglial tumors were AO tumors with $I D H$ mutant status and $1 \mathrm{p} / 19 \mathrm{q}$ codeletions in the latest WHO classification. ${ }^{15}$ In fact, $71 \%$ of $\mathrm{AO}$ tumors in our cohort were $I D H$ mutants with $1 \mathrm{p} / 19 \mathrm{q}$ codeletions. We are now collecting new data, and hope to report the analysis in the future.

\section{Conclusions}

Volumetric analysis using intraoperative MRI in patients with WHO Grade III gliomas at a single institute was performed. Our data indicated that T2-EOR has a strong correlation with survival in patients with AA and AOA, but not in patients with AO. In addition, T2-EOR $\geq$
53\% was associated with improved survival. Further investigation of other patient populations will be necessary to validate the effect of T2-EOR on patients with WHO Grade III gliomas.

\section{Acknowledgments}

We extend special thanks to Drs. Manabu Tamura, Atsushi Fukui, Yoshiyuki Konishi, and Takashi Komori for providing valuable supervision and help with the clinical work and data analysis. This research is partially supported by the Research and Development of Advanced Medical Devices and Systems to Achieve the Future of Medicine/Development of a Smart Treatment Chamber for the Improvement of Both Medical Safety and Efficiency from the Japan Agency for Medical Research and Development (AMED).

\section{References}

1. Beiko J, Suki D, Hess KR, Fox BD, Cheung V, Cabral M, et al: IDH1 mutant malignant astrocytomas are more amenable to surgical resection and have a survival benefit associated with maximal surgical resection. Neuro Oncol 16:81-91, 2014

2. Belhawi SMK, Hoefnagels FWA, Baaijen JC, Aliaga ES, Reijneveld JC, Heimans JJ, et al: Early postoperative MRI overestimates residual tumour after resection of gliomas with no or minimal enhancement. Eur Radiol 21:1526-1534, 2011

3. Chaichana KL, Jusue-Torres I, Navarro-Ramirez R, Raza SM, Pascual-Gallego M, Ibrahim A, et al: Establishing percent resection and residual volume thresholds affecting survival and recurrence for patients with newly diagnosed intracranial glioblastoma. Neuro Oncol 16:113-122, 2014

4. Chaichana KL, Kosztowski T, Niranjan A, Olivi A, Weingart JD, Laterra J, et al: Prognostic significance of contrastenhancing anaplastic astrocytomas in adults. J Neurosurg 113:286-292, 2010

5. Fukui A, Muragaki Y, Saito T, Maruyama T, Nitta M, Ikuta S, et al: Volumetric analysis using low-field intraoperative magnetic resonance imaging for 168 newly diagnosed supratentorial glioblastomas: effects of extent of resection and residual tumor volume on survival and recurrence. World Neurosurg 98:73-80, 2017

6. Glass J, Hochberg FH, Gruber ML, Louis DN, Smith D, Rattner B: The treatment of oligodendrogliomas and mixed oligodendroglioma-astrocytomas with PCV chemotherapy. J Neurosurg 76:741-745, 1992

7. Grabowski MM, Recinos PF, Nowacki AS, Schroeder JL, Angelov L, Barnett GH, et al: Residual tumor volume versus extent of resection: predictors of survival after surgery for glioblastoma. J Neurosurg 121:1115-1123, 2014

8. Iseki H, Nakamura R, Muragaki Y, Suzuki T, Chernov M, Hori T, et al: Advanced computer-aided intraoperative technologies for information-guided surgical management of gliomas: Tokyo Women's Medical University experience. Minim Invasive Neurosurg 51:285-291, 2008

9. Keles GE, Chang EF, Lamborn KR, Tihan T, Chang CJ, Chang SM, et al: Volumetric extent of resection and residual contrast enhancement on initial surgery as predictors of outcome in adult patients with hemispheric anaplastic astrocytoma. J Neurosurg 105:34-40, 2006

10. Kim W, Liau LM: IDH mutations in human glioma. Neurosurg Clin N Am 23:471-480, 2012

11. Kubben PL, ter Meulen KJ, Schijns OEMG, ter Laak-Poort MP, van Overbeeke JJ, van Santbrink H: Intraoperative MRIguided resection of glioblastoma multiforme: a systematic review. Lancet Oncol 12:1062-1070, 2011

12. Lacroix M, Abi-Said D, Fourney DR, Gokaslan ZL, Shi W, DeMonte F, et al: A multivariate analysis of 416 patients with 
glioblastoma multiforme: prognosis, extent of resection, and survival. J Neurosurg 95:190-198, 2001

13. Li YM, Suki D, Hess K, Sawaya R: The influence of maximum safe resection of glioblastoma on survival in 1229 patients: can we do better than gross-total resection? J Neurosurg 124:977-988, 2016

14. Louis DN, Ohgaki H, Wiestler OD, Cavenee WK: WHO Classification of Tumours of the Central Nervous System, ed 4. Lyon: IARC Press, 2007

15. Louis DN, Ohgaki H, Wiestler OD, Cavenee WK: WHO Classification of Tumours of the Central Nervous System, ed 4, revised. Lyon: IARC Press, 2016

16. Mohammadi AM, Sullivan TB, Barnett GH, Recinos V, Angelov L, Kamian K, et al: Use of high-field intraoperative magnetic resonance imaging to enhance the extent of resection of enhancing and nonenhancing gliomas. Neurosurgery 74:339-350, 2014

17. Muragaki Y, Iseki H, Maruyama T, Tanaka M, Shinohara C, Suzuki T, et al: Information-guided surgical management of gliomas using low-field-strength intraoperative MRI. Acta Neurochir Suppl 109:67-72, 2011

18. Nitta M, Muragaki Y, Maruyama T, Ikuta S, Komori T, Maebayashi K, et al: Proposed therapeutic strategy for adult low-grade glioma based on aggressive tumor resection. Neurosurg Focus 38(1):E7, 2015

19. Nomiya T, Nemoto K, Kumabe T, Takai Y, Yamada S: Prognostic significance of surgery and radiation therapy in cases of anaplastic astrocytoma: retrospective analysis of 170 cases. J Neurosurg 106:575-581, 2007

20. Nuño M, Birch K, Mukherjee D, Sarmiento JM, Black KL, Patil CG: Survival and prognostic factors of anaplastic gliomas. Neurosurgery 73:458-465, 2013

21. Ostrom QT, Gittleman H, Fulop J, Liu M, Blanda R, Kromer $C$, et al: CBTRUS Statistical Report: Primary Brain and Central Nervous System Tumors Diagnosed in the United States in 2008-2012. Neuro Oncol 17 (Suppl 4):iv1-iv62, 2015

22. Padwal JA, Dong X, Hirshman BR, Hoi-Sang U, Carter BS, Chen CC: Superior efficacy of gross total resection in anaplastic astrocytoma patients relative to glioblastoma patients. World Neurosurg 90:186-193, 2016

23. Pala A, Brand C, Kapapa T, Hlavac M, König R, Schmitz $\mathrm{B}$, et al: The value of intraoperative and early postoperative magnetic resonance imaging in low-grade glioma surgery: a retrospective study. World Neurosurg 93:191-197, 2016

24. Pessina F, Navarria P, Cozzi L, Ascolese AM, Simonelli M, Santoro A, et al: Value of surgical resection in patients with newly diagnosed Grade III glioma treated in a multimodal approach: surgery, chemotherapy and radiotherapy. Ann Surg Oncol 23:3040-3046, 2016

25. Puduvalli VK, Hashmi M, McAllister LD, Levin VA, Hess KR, Prados M, et al: Anaplastic oligodendrogliomas: prognostic factors for tumor recurrence and survival. Oncology 65:259-266, 2003

26. Saito T, Maruyama T, Muragaki Y, Tanaka M, Nitta M, Shinoda J, et al: ${ }^{11} \mathrm{C}$-methionine uptake correlates with combined $1 \mathrm{p}$ and $19 \mathrm{q}$ loss of heterozygosity in oligodendroglial tumors. AJNR Am J Neuroradiol 34:85-91, 2013

27. Saito T, Muragaki Y, Maruyama T, Komori T, Tamura M, Nitta M, et al: Calcification on CT is a simple and valuable preoperative indicator of $1 \mathrm{p} / 19 \mathrm{q}$ loss of heterozygosity in supratentorial brain tumors that are suspected grade II and III gliomas. Brain Tumor Pathol 33:175-182, 2016

28. Sanai N, Berger MS: Glioma extent of resection and its impact on patient outcome. Neurosurgery 62:753-764, 264266, 2008

29. Sanai N, Polley MY, McDermott MW, Parsa AT, Berger MS: An extent of resection threshold for newly diagnosed glioblastomas. J Neurosurg 115:3-8, 2011

30. Senft C, Bink A, Franz K, Vatter H, Gasser T, Seifert V: Intraoperative MRI guidance and extent of resection in glioma surgery: a randomised, controlled trial. Lancet Oncol 12:997-1003, 2011

31. Shirai K, Suzuki Y, Okamoto M, Wakatsuki M, Noda SE, Takahashi T, et al: Influence of histological subtype on survival after combined therapy of surgery and radiation in WHO grade 3 glioma. J Radiat Res (Tokyo) 51:589-594, 2010

32. Smith JS, Chang EF, Lamborn KR, Chang SM, Prados MD, Cha S, et al: Role of extent of resection in the long-term outcome of low-grade hemispheric gliomas. J Clin Oncol 26:1338-1345, 2008

33. Tortosa A, Viñolas N, Villà S, Verger E, Gil JM, Brell M, et al: Prognostic implication of clinical, radiologic, and pathologic features in patients with anaplastic gliomas. Cancer 97:1063-1071, 2003

34. Vuorinen V, Hinkka S, Färkkilä M, Jääskeläinen J: Debulking or biopsy of malignant glioma in elderly people - a randomised study. Acta Neurochir (Wien) 145:5-10, 2003

35. Wang Y, Wang K, Wang J, Li S, Ma J, Dai J, et al: Identifying the association between contrast enhancement pattern, surgical resection, and prognosis in anaplastic glioma patients. Neuroradiology 58:367-374, 2016

\section{Disclosures}

The authors report no conflict of interest concerning the materials or methods used in this study or the findings specified in this paper.

\section{Author Contributions}

Conception and design: Muragaki. Acquisition of data: Muragaki, Fujii, Maruyama, Nitta, Ikuta. Analysis and interpretation of data: Muragaki, Fujii, Maruyama, Nitta, Saito, Ikuta. Drafting the article: Fujii. Critically revising the article: Muragaki, Saito. Reviewed submitted version of manuscript: Muragaki, Maruyama, Nitta, Iseki, Hongo, Kawamata. Approved the final version of the manuscript on behalf of all authors: Muragaki. Statistical analysis: Fujii, Ikuta. Administrative/technical/material support: Muragaki. Study supervision: Muragaki, Iseki, Hongo, Kawamata.

\section{Correspondence}

Yoshihiro Muragaki, Faculty of Advanced Techno-Surgery, Institute of Advanced Biomedical Engineering and Science, Tokyo Women's Medical University, 8-1 Kawada-cho, Shinjuku-ku, Tokyo 162-8666, Japan. email: ymuragaki@twmu.ac.jp. 